\title{
Development and Evaluation of Controlled-Release Bilayer Tablets Containing Microencapsulated Tramadol and Acetaminophen
}

\author{
MA Naeem*, A Mahmood, SA Khan and Z Shahiq \\ Faculty of Pharmacy \& Alternative Medicine, The Islamia University of Bahawalpur, Punjab, Pakistan.
}

\begin{abstract}
Purpose: To develop and characterize bilayer tablet formulations of tramadol $\mathrm{HCl}(\mathrm{TmH})$ and acetaminophen $(A A P)$ microparticles.

Methods: Coacervation via temperature change was the encapsulated method used for the preparation of the microparticles, with ethyl cellulose (EC) of medium viscosity as the polymer for extending drug release. The microparticles of the two drugs were prepared separately and then compressed into bilayer tablets. The physicochemical compatibility and stability of the tablets were determined by Fourier transform infrared spectroscopy (FTIR), x-ray diffractometry (XRD), differential scanning calorimetry (DSC) and thermogravimetric analysis (TGA) while their mechanism and pattern of drug release were assessed by applying Higuchi, Zero order, First order and Korsmeyer-Peppas kinetic models. Bilayer tablets were subjected to accelerated stability studies for three months.

Results: FTIR, XRD, DSC and TGA data for the formulations indicate good compatibility and stability. Furthermore, accelerated stability studies confirmed the stability of the formulations. Controlled drug release from the microparticles and bilayer tablets was observed for $8 h$ and $12 h$, respectively. The Higuchi model produced the best fit, with regard to release profile, for both drugs, with correlation coefficient $\left(R^{2}\right)$ of 0.966 and 0.960 for $A A P$ and $T m H$, respectively.

Conclusion: Microencapsulated TmH and AAP can be developed into suitable bilayer tablets that are stable and capable of releasing the drugs over $12 \mathrm{~h}$.
\end{abstract}

Keywords: Acetaminophen; Tramadol; Ethyl cellulose; Microparticles; Bilayer tablets; Kinetic models. 


\section{INTRODUCTION}

Acetaminophen (AAP) is an odourless, slightly bitter, white crystalline powder. It is soluble in organic solvents such as methanol and ethanol but slightly soluble in water and ether. It is an analgesic and antipyretic drug that is used for the relief of fever, headache, and other moderate aches and pains [1]. AAP reduces production of prostaglandins, which are involved in pain and fever processes, by inhibiting the cyclooxygenase enzyme (COX-3) $[2,3]$.

Tramadol hydrochloride $(\mathrm{TmH})$ is a centrally acting analgesic having both opioid and nonopioid effects. TmH acts as opiate agonists, through selective binding to the $\mu$-opioid receptor, and weak inhibition of norepinephrine and serotonin uptake [4]. It is administered when non-steroidal antiinflammatory drugs fail to mitigate pain. It is readily absorbed after oral administration. Its half-life is $6.3 \mathrm{~h}[5]$.

Ethyl cellulose (EC), an ethyl ether of cellulose, is a long chain polymer of $\beta$ anhydro-glucose units joined together by acetal linkages. Ethyl cellulose is an inert hydrophobic polymer and is essentially odourless, tasteless, colorless, non-toxic, biocompatible and soluble in a wide range of organic solvents $[6,7]$.

Although a conventional solid dosage combination of $\mathrm{TmH}$ and AAP has been approved by the United States Food and Drug Administration (FDA) for use, patients still have to take the conventional tablets $3-4$ times a day. To improve patient's compliance, a controlled-released combination was developed and characterized in this study for its physical and chemical stability as well as for release characteristics.

\section{EXPERIMENTAL}

\section{Materials}

Tramadol hydrochloride (Ali Gohar Pharmaceuticals, Pakistan), acetaminophen (Neutro Pharma, Pakistan), methanol (Merck, Germany), ethyl cellulose (22 cP, BDH Chemicals Ltd, Poole, UK), cyclohexane (Merck, Germany) and n-hexane (Merck, Germany) were among the materials used in the study.

\section{Preparation of microparticles}

Microencapsulation, based on phase separation by temperature change was employed to formulate separate microparticles of $\mathrm{TmH}$ and AAP. In this technique, Ethyl cellulose (EC, $1 \mathrm{~g}$ ) was first dissolved in cyclohexane $(50 \mathrm{ml})$ by heating the mixture to $80{ }^{\circ} \mathrm{C}$ and then $\mathrm{TmH}(1 \mathrm{~g})$ was dispersed in it using a magnetic stirrer at 700 rpm. Phase separation was achieved through rapid temperature reduction by transferring the flask containing the mixture to an ice-bath with continuous stirring. The resulting microparticles, after washing with distilled water $(20 \mathrm{ml})$ and then with $\mathrm{n}$-hexane $(20 \mathrm{ml})$, were dried at $40{ }^{\circ} \mathrm{C}$ in an oven (Mammert, Germany). The dried microparticles were stored in an air-tight, amber-coloured glass container. Microparticles of AAP (EC: AAP ratio, 1:1) were similarly produced and stored.

\section{Morphological studies}

Morphological characteristics of the microparticles were studied by scanning electron microscopy (Hitachi, S $3000 \mathrm{H}$, Japan). The microparticles were dotted on an adhesive tape attached to an aluminum stub and excess microparticles were detached. To render the particles electrically conductive, the stub sputter was coated with gold using a vacuum evaporator. The coated microparticles were viewed at $25 \mathrm{kV}$. 


\section{Drug loading}

$\mathrm{TmH}$ content of microparticles was determined by dissolving $41.5 \mathrm{mg}$ of the microparticles in $8 \mathrm{ml}$ of methanol in order to remove the EC coating around the microparticles. Distilled water $(20 \mathrm{ml})$ was added to precipitate EC and heated to evaporate the methanol. The precipitated EC was filtered off and the volume of the drug was made to $100 \mathrm{ml}$ with water. Aliquot of this solution was taken, diluted to $100 \mathrm{ml}$ and analyzed by UV-spectrophotometry (Shimadzu 1601, Japan) at $270 \mathrm{~nm}$. The absorbance of standard solutions of the pure drug was also determined, from which the drug loading of the microparticles was derived. The same procedure was adopted for AAP except that absorbance measurements were made at a wavelength of $245 \mathrm{~nm}$.

\section{Preparation of TmH-AAP bilayer tablets}

Bilayer tablets of $\mathrm{TmH}$ and AAP (equivalent to $100 \mathrm{mg}$ and $500 \mathrm{mg}$, respectively) were prepared by direct compression of their microparticles [8]. The microparticles of each drug were first blended with $0.75 \%$ each of talc and magnesium stearate prior to a double compression process with a single punch tablet machine (Emmay, Pakistan). First AAP microparticles were compressed at low pressure and then $\mathrm{TmH}$ microparticles were compressed over them.

\section{In-vitro drug release of microparticles and bilayer tablets}

In-vitro dissolution tests on $\mathrm{TmH}$ and $\mathrm{AAP}$ microparticles (165 mg and $531 \mathrm{mg}$, respectively) were carried out separately using USP apparatus-II (Pharma Test, Germany) at $50 \mathrm{rpm}$ [9]. The dissolution medium $(900 \mathrm{ml})$ consisted of $0.1 \mathrm{M}$ hydrochloric acid ( $\mathrm{pH} 1.0)$ for the first $2 \mathrm{~h}$ and then replaced with phosphate buffer ( $\mathrm{pH}$ 6.8) from the $3^{\text {rd }}$ to the $8^{\text {th }}$ hour to simulate gastrointestinal conditions. The temperature of the dissolution media was maintained at 37 $\pm 0.5^{\circ} \mathrm{C}$ while sink conditions were attained by enclosing the microparticles in stainless steel sieves, placed at the bottom of dissolution vessel. A $5 \mathrm{ml}$ sample of the dissolution fluid was collected at regular intervals with an automated sample collector after filtering through a $10 \mu \mathrm{m}$ sinter filter and replaced with an equal volume of fresh dissolution medium stored at $37 \pm 0.5^{\circ} \mathrm{C}$. The withdrawn samples were analyzed at 270 and $245 \mathrm{~nm}$ for $\mathrm{TmH}$ and AAP, respectively, using a double-beam UV/visible spectrophotometer (Shimadzu 1601, Japan). The apparatus, methodology and specifications adopted for the dissolution test on bilayer tablets were the same as that for microparticles except that the test was extended to $12 \mathrm{~h}$. All the tests were conducted in triplicate.

\section{Evaluation of tablet hardness, friability and weight variation}

The hardness of the tablets was determined with an automatic hardness tester (Curio, Pakistan). Ten tablets were used in each test and the mean hardness was calculated. The friability of the tablets was evaluated with a friabilator (Emmy, Pakistan). Twenty tablets were weighed prior to placing them in the friabilator chamber and at the end of the test, their weight was also recorded. Finally, the loss in weight was calculated. For weight uniformity test, twenty tablets were randomly selected and weighed using a 'Class A' electronic weight balance (Precisa, Switzerland) and weight variation (\%) was calculated.

\section{Kinetic analysis of release data}

The dissolution data of the bilayer tablets were fitted to some kinetic models, namely, Zero order, First order, Higuchi [10], and Korsmeyer-Peppas [11,12] in order to determine $\mathrm{TmH}$ and AAP release patterns and mechanisms. 


\section{Accelerated stability studies}

Bilayer tablets were packed in air-tight amber glass wide-mouth bottles $(100 \mathrm{ml})$ with 25 30 tablets in each bottle, and then sealed with aluminum foil. The bottles were kept at $40 \pm 2{ }^{\circ} \mathrm{C} / 75 \pm 5 \% \mathrm{RH}$. Twenty one tablets (18 for dissolution and 3 for drug assay) were taken from each bottle at 1,2 and 3 months, and evaluated for stability by determining invitro release profile and drug content. Similarity factor $\left(f_{2}\right)$ was used to compare the difference of dissolution profile after regular intervals. According to FDA guidelines, $f_{2^{-}}$ values between 50 and 100 ensure equivalence of two sets of dissolution data.

$f_{2}=50 \log \left\{\left[1+1 / n \sum_{t=1}^{n}\left(R_{t}-T_{t}\right)^{2}\right]^{-0.5} \times 100\right\} \ldots$.

where $R_{t}$ and $T_{t}$ represent dissolution values of the reference and test products, respectively.

\section{X-ray diffractometry}

The crystalline behaviour of $\mathrm{TmH}$ and AAP, before and after encapsulation, was evaluated by X-ray powder diffractometer (Bruker D8 Discover, Germany) using a CuK alpha radiation source with Ni-filter. A scanning rate of $5^{0} \mathrm{~min}^{-1}$, tube voltage of 35 $\mathrm{KV}$ and current of $35 \mathrm{~mA}$ over a range of 8$60^{\circ}$ were used in measurement.

\section{Thermal analysis}

Differential scanning calorimetry and thermogravimetric analysis of the pure drugs and drug-loaded bilayer tablets were carried out simultaneously with simultaneous DSC/TGA analyzer (model SDT Q600). An amount $(4-5 \mathrm{mg})$ of the crushed bilayer tablets was placed in aluminum pans and sealed prior to the test. Measurement was performed at a rate $20{ }^{0} \mathrm{C} \mathrm{min}^{-1}$ under nitrogen flow of $25 \mathrm{ml} \min ^{-1}$ over a temperature range of 25 to $180{ }^{\circ} \mathrm{C}$. Indium was used for the calibration of the equipment.
Fourier transform infrared (FTIR)
spectroscopy

Drug-polymer interactions were assessed by FTIR spectroscopy. The spectra were recorded for $\mathrm{TmH}, \mathrm{AAP}$ and EC singly as well as for the drug-loaded bilayer tablets using FTIR Midac 2000, USA. Samples were prepared in $\mathrm{KBr}$ disks and the test was carried out at a resolution of $2 \mathrm{~cm}_{-1}^{-1}$ and a scanning range of $500-4000 \mathrm{~cm}^{-1}$.

\section{Data analysis}

One-way ANOVA Post-Hoc analysis (Duncan and Turkey) was used for regression analysis of drug release data derived from model equations with the aid of SPSS package, version 12.0. Statistical significance was set at $p \leq 0.05$. Taking into account the number of independent variables, $R^{2}$ was used to determine how well a regression model describes the release data. Mean \pm SD for drug loading of microparticles and hardness of tablets was calculated by using Microsoft Office Excel 2003.

\section{RESULTS}

\section{Shape and drug loading of microparticles}

Spherical microparticles with a smooth surface were observed. Mean drug loading (n =3) was $68.0 \pm 1.6$ and $74.0 \pm 2.3 \%$ for $\mathrm{TmH}$ and AAP, respectively.

\section{Tablet hardness, friability and weight variation}

Mean hardness, friability and weight variation of the tablets were $8 \pm 1.19 \mathrm{~kg}, \leq 0.05 \%$ and $\pm 0.2 \%$, respectively.

\section{In-vitro drug release}

Fig 1 shows the in vitro release profiles of the microparticles. After 8 h, 97.0 and $99.7 \%$ of AAP and $\mathrm{TmH}$, respectively, were released from the microparticles while 92.6 and $99.5 \%$ 
of $\mathrm{TmH}$ and $\mathrm{AAP}$, respectively, were released from the bilayer tablets after $12 \mathrm{~h}$.
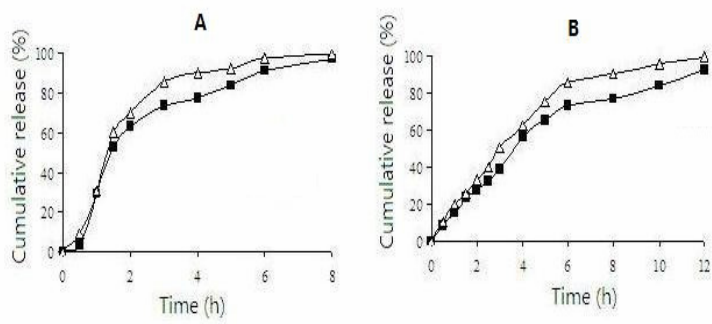

Figure 1: Drug release from $(A)$ microparticles and (B) bilayer tablets containing AAP $(\mathbf{-})$ and $\mathrm{TmH}(\Delta)$

\section{Drug-polymer interaction}

\section{$X$-ray diffractometry}

Fig 2 shows the crystallographic pattern of the drugs, polymer and tablet formulation. Every crystalline substance has a specific XRD pattern, which can be used for its detection, just like fingerprint. EC showed a single characteristic peak while $\mathrm{TmH}$, AAP and bilayer tablets presented multiple peaks.

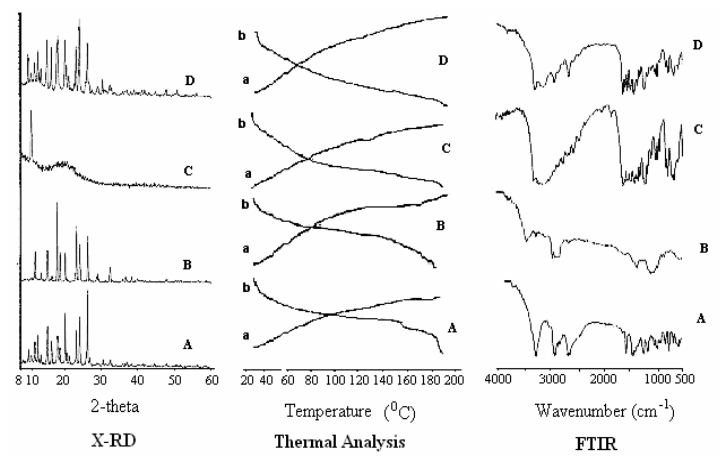

Figure 2: Diffractograms, thermograms and FTIR spectra of $\mathrm{TmH}(\mathrm{A})$, AAP (B), EC (C) and bilayer tablets (D). Note: For thermal analysis, $a=D S C$ thermogram and $b=$ TGA thermogram

\section{Thermal analysis}

Fig 2 also shows the data obtained by thermal analysis. The DSC thermograms showed endothermic peaks around 181 and $168{ }^{\circ} \mathrm{C}$ which indicate the melting point of
$\mathrm{TmH}$ and AAP, respectively. Similarly, an endothermic peak at $129{ }^{\circ} \mathrm{C}$ indicates the glass transition temperature ( $\mathrm{Tg}$ ) of EC. TGA thermograms of all the components and bilayer tablets showed downward shift which indicated loss of mass (due to both moisture loss and degradation) upon heating.

\section{FTIR spectroscopy}

In the FTIR spectrum of $\mathrm{TmH}$ (Fig 2), the characteristic peaks of aromatic ring stretching at $1600 \mathrm{~cm}^{-1}$, aliphatic $\mathrm{CH}$ stretching at $2900 \mathrm{~cm}^{-1}$, aromatic $\mathrm{CH}$ stretching at $3050 \mathrm{~cm}^{-1}$ and $\mathrm{OH}$ shoulders at $3300 \mathrm{~cm}^{-1}$, respectively, were seen. The FTIR spectra of AAP showed $\mathrm{C}-\mathrm{N}$ amide stretching peaks (1566, 1507 and 1560, 1513 $\mathrm{cm}^{-1}$ ) and $\mathrm{C}-\mathrm{C}$ aromatic stretching peaks (1614, 1507, 1442 and 1626, 1513, $1453 \mathrm{~cm}^{-}$ 1) [13]. EC displayed two main characteristic peaks, first at $3365 \mathrm{~cm}^{-1}$ due to stretching vibration of $\mathrm{OH}$ groups at carbon numbers 2, 3 and 6 , and a second peak at $3210 \mathrm{~cm}^{-1}$ due to $\mathrm{OH}$ bond at carbon numbers 1 and 4 .

\section{Stability studies}

Fig 3 shows the results for accelerated stability studies in terms of their effect on drug release. There was no significant difference $(p<0.05)$ between the release profiles of $\mathrm{TmH}$ and AAP after storage for 1 , 2 and 3 months. $f_{2}$ values were greater than 50 (80.98, 86.45, 86.02 for AAP and 79.56, 84.56 and 85.70 for $\mathrm{TmH}$, after 1,2 and 3 months, respectively, of stability studies) thus showing that no significant change in release patterns of both drugs occurred over the period of the stability studies. The drug content of the tablets was $100.2,98.0,96.8$ and $97.4 \%$ for $\mathrm{TmH}$, and 101.1, 98.7, 96.5 and $95.8 \%$ for AAP after 0, 1, 2 and 3 months, respectively, of stability studies, thus demonstrating that no significant degradation of the drugs took place over the period. 


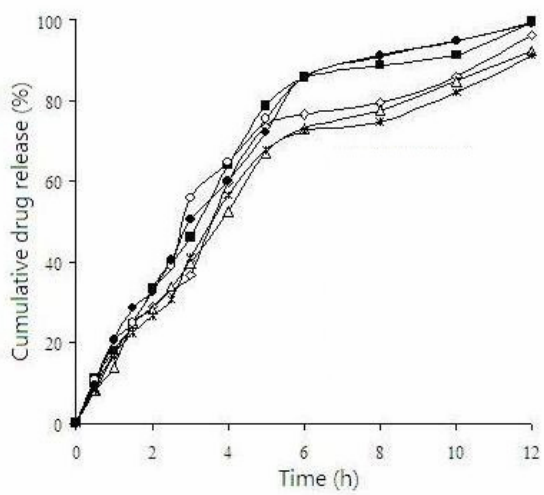

Figure 3: Accelerated stability studies - Drug release pattern of $\mathrm{TmH} / \mathrm{AAP}$ bilayer tablets after 1 month $(\diamond=\mathrm{AAP} ;=\mathrm{TmH}), 2$ months $(\Delta=\mathrm{AAP}$; 。 $=\mathrm{TmH})$, and 3 months $(\%=\mathrm{AAP} ; \bullet=\mathrm{TmH})$

\section{DISCUSSION}

\section{Drug release and kinetics}

Microparticles are small units and, therefore, drug would readily be released from them due to their large surface area. On the other hand, tablets are large compact masses of particles and sufficient time would be required for medium to penetrate into them because of their much smaller surface area [14]. The hardness of the tablets was kept high and constant in order to prevent disintegration during drug release. Furthermore, the release of both drugs was independent of $\mathrm{pH}$ of the dissolution medium used. $\mathrm{TmH}$ is a salt of basic drug and is soluble in acidic as well as in basic medium. AAP is unionized in $0.1 \mathrm{M} \mathrm{HCl}(\mathrm{pH} \mathrm{1.2)}$ and phosphate buffer ( $\mathrm{pH}$ 6.8).

The release patterns of $\mathrm{TmH}$ and AAP were predicted by application of zero-order, firstorder and Higuchi models while release mechanism was evaluated by the KorsmeyerPeppas model. Release constant (k) and regression coefficient $\left(R^{2}\right)$ for $\mathrm{TmH}$ and $\mathrm{AAP}$ release kinetics indicated that their release was best described by the Higuchi model, based on the highest linearity $\left(R^{2}=0.966\right.$ for $\mathrm{AAP}$ and $\mathrm{R}^{2}=0.960$ for $\mathrm{TmH}$ ); a close relationship with zero-order kinetics was also found $\left(R^{2}=0.910\right.$ for AAP and $R^{2}=0.881$ for $\mathrm{TmH}$ ). Korsmeyer-Peppas plot indicated an $n$ value of 0.65 for AAP and 0.61 for $\mathrm{TmH}$, both of which are indicative of an anomalous diffusion mechanism i.e., diffusion coupled with erosion. Thus, the release of both drugs was regulated by more than a single process $[15,16]$.

\section{Drug-polymer interaction}

\section{$X$-ray diffractometry}

The intensity of the diffraction peaks of $\mathrm{TmH}$ and AAP was reduced when the drugs were encapsulated in the polymer (EC), which indicates reduced crystallinity of $\mathrm{TmH}$ and AAP. The decrease in crystallinity indicates a change in the physical stability of TmH-AAPEC combination with the drugs showing increased amorphous nature [17]. The crystalline behavior of $\mathrm{TmH}$ and AAP was present in the spectra of bilayer tablets which mean both drugs remained intact and there was no interaction of drugs with polymer.

\section{Thermal analysis}

For the bilayer tablets, the lowering of $\mathrm{Tg}$ of EC to $118{ }^{\circ} \mathrm{C}$ and melting point of $\mathrm{TmH} 180$ ${ }^{0} \mathrm{C}$ was observed. This indicates a decrease in the stability of the drug in the tablet form, compared to the pure drug [18,19]. AAP showed no melting peak in the bilayer tablets, probably due to solvation in the solvent. The endothermic peaks of EC and $\mathrm{TmH}$ were unchanged in the bilayer tablets, thus indicating compatibility between the two compounds. The continuous downward trend of TGA thermograms suggests that mass loss was due to moisture/residual solvent loss and decomposition of the tablet materials.

\section{FTIR spectroscopy}

No new bands were detected in the FTIR spectra of bilayer tablets, indicating no chemical interaction between TmH-AAP-EC 
combinations. Therefore, both drugs were chemically stable in the tablet formulations. Physical conjugation of the polymer with the drugs was probably responsible for the prolonged-release characteristics of $\mathrm{TmH}$ and AAP.

\section{Stability of the bilayer tablets}

The data obtained from drug assay and stability studies, including the values of the similarity factor $\left(f_{2}\right)$ [[20] indicate that drug content of, and release from, the tablets were essentially unchanged after 3 months, suggesting that the tablets would be able to tolerate harsh storage conditions.

\section{CONCLUSION}

Coacervation via temperature change method can be employed for the development of bilayer tablet formulations of tramadol/acetaminophen combination, using ethyl cellulose as the encapsulating polymer for retarding drug release from the tableted microparticles. Prolonged release of up to 12 $\mathrm{h}$ was achieved thus making it feasible to attain reduced frequency of administration of the drug combination.

\section{ACKNOWLEDGEMENT}

We appreciate the Higher Education Commission of Pakistan (HEC) for promoting research into pharmaceuticals and providing financial support for this project.

\section{REFERENCES}

1. Richard $C D$, Edwin $K K$, Barry HR. Treatment of pain or fever with paracetamol (Acetaminophen) in the alcoholic patient: $A$ systematic review. Am. J. Therapeu. 2000; 7: 123-134.

2. Ruth L, Timothy DW, Ivana V, Jane AM. Cellular mechanisms of acetaminophen: role of cyclooxygenase. The FASEB J. 2005; 19: 635-637.

3. Botting RM. Mechanism of action of acetaminophen: is there a cyclooxygenase 3. Clin Infect Dis. 2000; 31: 202-210.

4. Valle M, Garrido MJ, Pavon JM, Calvo R, Troconiz IF. Pharmacokinetic pharmacodynamic modeling of the antinociceptive effects of main active metabolites of Tramadol, (b)-OdesmethylTramadol and (-)-O-desmethyl Tramadol, in rats. J Pharma. Exp Ther. 2000; 293: 646-653.

5. Raffa $R B$, Friderichs $E$, Reimann W, Shank RP, Codd EE, Vaught JL, Opioid and nonopioid components independently contribute to the mechanism of action of Tramadol, an 'atypical' opioid analgesic. J Pharmacol Exp Ther. 1992; 260: 275-285

6. Rowe RC, Sheskey RJ, Weller PJ. Handbook of Pharmaceutical Excipients. $4^{\text {th }}$ edn, 2003; $p$ 237.

7. Maarit T, Riitta S, Soili Pe, Hannu M, Jaa KH, Vesa $P L$, Petteri $P$. Enhanced film-forming properties for ethyl cellulose and starch acetate using n-alkenyl succinic anhydrides as novel plasticizers. Euro J Pharma Sci. 2003; 19: 363-371.

8. Sajeev C, Vinay G, Archna R, Saha $R N$, Oral controlled release formulation of diclofenac sodium by microencapsulation with ethylcellulose. J. Microencap. 2002; 19: $753-$ 760

9. Rao BP, $\square$ Geetha M, Purushothama N, Utpal S. Optimization and Development of Swellable Controlled Porosity Osmotic Pump Tablet for Theophylline. Trop J Pharm Res, 2009; 8 (3): 247-255

10. Higuchi T. Mechanism of sustained action medication, Theoretical analysis of rate of release of solid drugs dispersed in solid matrices. J Pharm Sci. 1963; 52: 1145-1149.

11. Korsmeyer RW, Gumy $R$, Doelker $E$, Buri $P$, Peppas NA. Mechanism of solute release from porous hydrophilic polymer. Int J Pharm. 1983; 15: 25-35.

12. Peppas NA. Analysis of Fickian and non-Fickian drug release from polymers. Pharm Acta Helv. 1985; 60: 110-111.

13. Kiran $S B$, Ravindra $S D$, Bhaskar $C$, Anant $P$, Shivajirao SK. Effect of Oppositely Charged Polymer and Dissolution Medium on Swelling, Erosion, and Drug Release From Chitosan Matrices. AAPS PharmSciTech 2007; 8: Article 44.

14. Nokhodchi, Zakeri-Milani $P$, Valizadeh $H$, HassanZadeh D. Evaluation of microspheres of acetyl salicylic acid prepared with cellulose acetate phthalate, ethycellulose or their mixtures by an emulsion non-solvent addition technique. Ars Pharmac. 2002; 43: 135-147.

15. Niuris $A$, Inmaculada $A$, Carlos $P$, Angeles $H$. Tramadol Release from a Delivery System Based on Alginate-Chitosan Microspheres. Macromol Biosci. 2003; 3: 546-551.

16. Apurba SA, Atiqul HP, Dilasha S, Golam K, Reza-ul Jalil. Investigation of In vitro Release Kinetics of Carbamazepine from Eudragit ${ }^{\circledR}$ RS $P O$ and RL PO Matrix Tablets. Trop J Pharma Res. 2009; 8 (2): 145-152. 
Naeemet al

17. Basu SK, Adhiyaman R. Preparation and Characterization of Nitrendipine-loaded Eudragit RL 100 Microspheres Prepared by an Emulsion-Solvent Evaporation Method. Trop J Pharm Res. 2008; 7 (3): 1033-1041.

18. Zhaoyang $W$, Xiaona $H$, Zhengzhou $M$, Ruirong $Y$, Yangqing $M$, David EF. Synthesis and Characterization of Biodegradable Poly (lactic acid-coglycine) via Direct Melt Copolymerization. Iran Poly J. 2008; 17: 791-798.

19. Emami J, Varshosaz J, Saljoughian $N$. Development and evaluation of controlledrelease buccoadhesive verapamil hydrochloride tablets. DARU 2008; Vol. 16: No. 2.

20. Moore JW, Flanner HH. Mathematical comparison of dissolution profiles, Pharm Tech. 1996; 20: 64-74. 